\title{
Force sensing on cells and tissues by atomic force microscopy
}

\author{
Hatice Holuigue ${ }^{1}$, Ewelina Lorenc ${ }^{1}$, Matteo Chighizola ${ }^{1}$, Carsten Schulte ${ }^{1}$, Luca Varinelli²,
} Marcello Deraco ${ }^{3}$, Marcello Guaglio ${ }^{3}$, Manuela Gariboldi², Alessandro Podestà ${ }^{1,}$

${ }^{1}$ CIMAINA and Dipartimento di Fisica "Aldo Pontremoli", Università degli Studi di Milano, via Celoria 16, 20133 Milano, Italy.

${ }^{2}$ Department of Research, Fondazione IRCCS Istituto Nazionale Tumori, via G. Venezian 1, 20133 Milan, Italy.

${ }^{3}$ Peritoneal Surface Malignancies Unit, Colon and Rectal Surgery, Fondazione IRCCS Istituto Nazionale Tumori, via G. Venezian 1, 20133, Milan, Italy.

*Corresponding author's e-mail: alessandro.podesta@mi.infn.it

\begin{abstract}
Biosensors are aimed to detect tiny physical and chemical stimuli in biological systems. Physical forces are ubiquitous, being implied in all cellular processes, including cell adhesion, migration, and differentiation. Given the strong interplay between cells and their microenvironment, the extracellular matrix $(E C M)$, the structural and mechanical properties of the ECM play an important role in the transmission of external stimuli to single cells within the tissue. Vice versa, also cells themselves use self-generated forces to probe the biophysical properties of the ECM. ECM mechanics influences cell fate, regulates tissue development and show peculiar features in health and disease conditions of living organisms. Force sensing in biological systems is therefore crucial to dissect and understand complex biological processes, such as mechanotransduction.
\end{abstract}

Atomic Force Microscopy (AFM), which can both sense and apply forces at the nanoscale, with sub-nanoNewton sensitivity, represents an enabling technology and a crucial experimental tool in biophysics and mechanobiology.

In this work, we report on the application of AFM to study of biomechanical fingerprints of different components of biological systems, such as the ECM, the whole cell, and cellular components, like the nucleus and the glycocalyx. We show that physical observables like the (spatially resolved) Young's modulus of elasticity of ECMs or cells, and the effective thickness and stiffness of the glycocalyx, can be quantitatively characterised by AFM. Their modification can be correlated to changes of the microenvironment, physio-pathological conditions, or gene regulation. 


\section{Introduction}

Through the last years, there has been a growing interest in studying the physical properties of biological samples, such as tissues, single cells and their microenvironment, to better understand how they change during the progression of diseases, such as cancer [1-3], and how they influence each other in their mutual interaction [4-11]. The extracellular matrix (ECM), which is a fundamental component of the cell microenvironment, is a ubiquitous acellulated component present in all tissues, constituted by molecules that are secreted by cells and assembled to form specific insoluble components; the ECM plays a fundamental role as a scaffold for cell growth, in the regulation of cell-cell and cell-matrix signalling, also affecting cell mechanics mainly through the remodelling of the cytoskeleton, and determines cell fate $[4,5,12-16]$. There is a reciprocal interaction between the ECM and the cells, allowing the active modification of the ECM structure and composition, which affects its mechanical properties as well $[9,17-19]$. Between the cell membrane and the cell microenvironment, an important physical layer is located: the glycocalyx, also known as the pericellular matrix; it is a surface brush layer that is present on every cell, and made of glycoproteins and polysaccharides $[16,20]$. As the first contact interface between the cell and its microenvironment, the glycocalyx plays an important role in their mutual interactions [21]. The glycocalyx acts as a water reservoir, helps in the transport of metabolites and control of the signalling molecules $[20,22]$, regulates integrin clustering and reinforces focal adhesion [23-25]. The characterisation of the glycocalyx thickness in relation to different pathological state of the cell would help to understand the communication between cells and the ECM [26]. More importantly, also a link has been demonstrated between glycocalyx and cancer: tumoral cells tend to show a wider distribution of glycocalyx chain lengths compared to normal ones [27]; moreover, their bulky composition seems to favour the metastatic spread [28-30]. The physical characterisation of glycocalyx has a good potential in cancer research, both as a cancer biomarker $[20,25,27,30,31]$ and as a therapeutic target, since the reduction or degradation of the glycocalyx has been reported to reduce the cell migration and suppress cell growth [32,33].

The study of the mechanical properties of cells and tissues in the context of health and disease implies the need for reliable instrumentation and methods. Atomic Force Microscopy (AFM), which is able to both sensing and applying forces at the nanoscale, with sub-nanoNewton sensitivity, represents an enabling technology and a crucial experimental tool in biophysics and biomechanics [34-37].

In AFM, an elastic cantilever with an intrinsic spring constant $k$ in the range $0.05-50 \mathrm{~N} / \mathrm{m}$ is used as both a force sensor and a force transducer (Figure 1A). The surface force $F$ is applied on the cantilever, typically at its end, where a micro-tip with a radius of curvature of typically $2-100 \mathrm{~nm}$ radius is located. The force induces a vertical cantilever deflection $z=F / k_{\text {eff, }}$, that is typically measured by an optical beam deflection apparatus [38-40] (Figure 1), or in some cases by an interferometer [41]. In the above equation, an effective spring constant $k_{\text {eff }}$ is used, rather than the intrinsic one, to account for specific features of the loading configuration, such as the cantilever mounting angle $\theta$ (usually $\theta=10^{\circ}-15^{\circ}$ ), the tip height, the loading point position etc[42]. In the simplest case of a negligibly small tip at the very end of the cantilever, $k_{\text {eff }}=k / \cos ^{2}(\theta)$. 

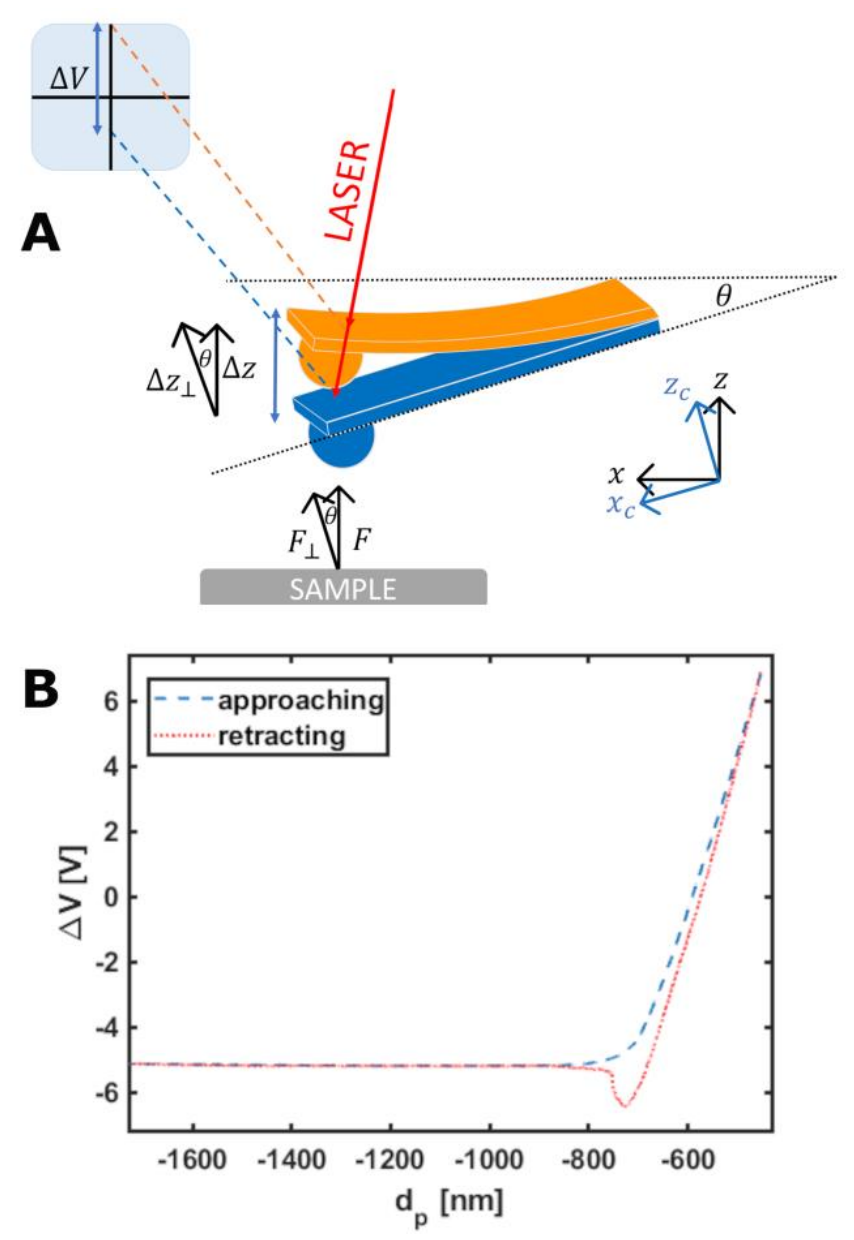

Figure 1. (A) Scheme of the optical beam deflection (OBD) system. The vertical displacement of the cantilever induced by the sensing of a force $F$ perpendicular to the sample surface is detected on a segmented photodiode as a raw voltage signal $\Delta \mathrm{V}$. The cantilever is typically mounted at an angle $\theta$ with respect to the sample surface. (B) $A$ raw force curve, representing the photodiode output $\Delta \mathrm{V}$ as a function of the z-piezo displacement $d_{p}$. Both the approaching and retracting branches of the curve are shown.

A small spring constant provides high force sensitivity, meaning that a small force produces a large, easily measurable, deflection. A lower limit to the measurable deflection (and therefore to the measurable force), is set by the thermal noise $z_{\text {th }}$ of the cantilever, which can be estimated from the equipartition theorem as $\mathrm{z}_{t h}=\sqrt{k_{B} T / k}, k_{B}$ and $T$ being the Boltzmann constant and the absolute temperature, respectively $[43,44]$. The minimum, thermal noise limited detectable force that can be measured dynamically with an instrumental bandwidth $B W$ is $\mathrm{F}_{t h, \min }=\sqrt{4 k_{B} T b B W}$, where $\mathrm{b}$ is the damping coefficient (the proportionality factor between the tip velocity and the viscous force). Equivalently, since $\left.b=k /\left(2 \pi \mathrm{f}_{R} Q\right)\right), \mathrm{F}_{t h \text {,min }}=$ $\sqrt{2 k_{B} T k B W /\left(\pi \mathrm{f}_{R} Q\right)}, Q$ and $f_{R}$ being the quality factor and the resonance frequency of the cantilever, respectively; similar expressions for the minimum force gradients can be obtained $[45,46]$.

Beside measuring the tip-sample interaction force with sub-nN sensitivity, AFM allows to reconstruct the tip-sample distance corresponding to the force measurements, which translates into a sample deformation after contact is established [47]. The possibility of 
measuring force vs distance, spatially resolved with $\mathrm{nm}$ resolution, assigns AFM a leading position as force (bio)sensing technique. AFM is at present an enabling technology and a crucial experimental tool in biophysics and biomechanics, allowing both force spectroscopy and nanomechanical characterization of biologically relevant interfaces and systems.

In this work, we report on the application of AFM to the study of biomechanical fingerprints of several components of biological systems, like the ECM, the whole cell, and cellular components, like the nucleus and the glycocalyx. We show that physical observables like the (spatially resolved) Young's modulus of elasticity of ECMs or cells and the effective thickness and stiffness of the glycocalyx can be quantitatively characterised by AFM, and their modification can be correlated to changes of the microenvironment, physio-pathological conditions, or gene regulation. In particular, we carried out three representative experiments from the microscale to the nanoscale: mechanics on healthy and neoplastic decellularized tissue from one patient with peritoneal metastasis; mechanics of three bladder cancer cell lines who are representative of the progression of urothelial bladder cancer and eventually glycocalyx characterisation of those cell lines.

\section{Material and methods}

\section{Sample preparation}

\section{Human tissue}

Peritoneal tissue was collected from one patient with peritoneal metastatic colorectal carcinoma (CRCPM) who underwent surgical resection at the Peritoneal Malignancies Unit of IRCCS Foundation, Istituto Nazionale dei Tumori di Milano, Milan, Italy. The patient was staged according to the World Health Organization (WHO) classification. The study was approved by the Institutional review board (249/19) and was conducted in accordance with the Declaration of Helsinki, 2009. Written informed consent was acquired.

Omentum-derived CRCPM lesion, and apparently normal tissue ( $>10 \mathrm{~cm}$ from the metastatic lesion) were harvested. Tissues were frozen in liquid nitrogen and used to develop the decellularized ECMs.

\section{Decellularized ExtraCellular Matrices}

Decellularized extracellular matrices were obtained from the omentum fold of human peritoneum from a patient with metastases derived from colorectal cancer. The decellularization was performed as in Genovese et. al. [48]. The success of the decellularization procedure was already verified in the work from Varinelli et. al. [49]. The ECM samples were embedded in optical cutting compound (OCT) and frozen in 2-propanol, kept in liquid nitrogen bath.

Slices of approximately $100 \mu \mathrm{m}$ thickness were cut with a microtome (Leica) and attached to positively charged poly-lysine -coated glass coverslips (ThermoFisher Scientific) exploiting the electrostatic interactions. The samples were stored at $-4^{\circ} \mathrm{C}$ until AFM analysis.

Cells

Three human bladder cancer cell lines of different grade (a marker of invasiveness), kindly provided by Dr. M. Alfano (San Raffaele Hospital, Milano), were used (see Table 1). The cell 
lines were cultured in RPMI medium containing $2 \mathrm{mM}$ L-glutamine supplemented with $10 \%$ FBS, $1 \%$ penicillin/streptomycin and $1 \%$ amphotericin and grown in an incubator at $37^{\circ} \mathrm{C}$ and $5 \% \mathrm{CO}_{2}$ (Galaxy S, RS Biotech). All reagents and material were from Sigma Aldrich, if not stated otherwise.

For AFM measurements, the cells were plated the day before on glass bottom petri dishes ( $\varnothing$ $40 \mathrm{~mm}$ Willco Wells) coated with poly-L-lysine (0.1\% w/v for $30 \mathrm{~min}$ at RT) in the RPMI medium without phenol red, as it can cause damage to the AFM probe holder.

\begin{tabular}{c|ccc} 
Cell line & Specie / Organ & Morphology & Tumour \\
\hline $\boldsymbol{R}$ T4 & Human bladder & Epithelial & $\begin{array}{c}\text { Papilloma, transitional cell } \\
\text { (grade 1) }\end{array}$ \\
$\boldsymbol{R}$ T112 & Human Bladder & Epithelial & $\begin{array}{c}\text { Papilloma, transitional cell } \\
\text { (grade 2) }\end{array}$ \\
T24 & Human bladder & Epithelial & $\begin{array}{c}\text { Carcinoma, transitional cell } \\
\text { (grade 3) }\end{array}$
\end{tabular}

Table 1: Characteristics of the bladder cancer cell line used in this work.

\section{Force sensing with the AFM}

All the experiments have been performed using a Bioscope Catalyst AFM (Bruker) mounted on top of an inverted microscope optical microscope (Olympus X71). The system was isolated from the noise using an active antivibration base (DVIA-T45, Daeil Systems) placed inside an acoustic enclosure (Schaefer, Italy). Living cells were measured using a thermostatic fluid cell, with the temperature of the medium kept at $37^{\circ} \mathrm{C}$ by a temperature controller (Lakeshore 331, Ohio, USA). The measurements on ECMs were performed at room temperature in a droplet of PBS confined on the glass slide using a hydrophobic pen.

Homemade colloidal probe were produced by attaching borosilicate glass or soda lime spheres to rectangular tipless cantilevers (NanoandMore TL-FM and MikroMasch $\mathrm{HQ}: \mathrm{CSC} 38 /$ Tipless/No Al); both production of the probes and characterisation of their radius were performed according to custom procedures [50]. Different sphere radii $R$ and spring constants $k$ of the probe were selected according to the needs of each experiment (Table 2 ).

\begin{tabular}{c|cc} 
Experiment & Colloidal probe radius $(\mu \mathrm{m})$ & Spring constant $(\mathbf{N} / \mathbf{m})$ \\
\hline Mechanics of ECM & 20 & 5 \\
Mechanics of cells & 5 & 0.01 \\
Glycocalyx characterisation & 5 & 0.01
\end{tabular}

Table 2: Radius and typical spring constant of the AFM probes used for every experiment. 
The raw output on an AFM force measurement consists of the raw deflection $\Delta \mathrm{V}$ of the cantilever, measured by the optical beam deflection (OBD) system in Volts units, as a function of the z-piezo displacement $d_{p}$, in $n m$ units (Figure 1A,B). Depending on the AFM system, the z-piezo can displace either the probe or the sample.

Exploiting two calibration parameters, the effective spring constant $k_{\text {eff }}(\mathrm{N} / \mathrm{m})$ and the deflection sensitivity $S$ (or inverse optical lever sensitivity invOLS, in units of $(V / \mathrm{nm})$, the raw deflection $\Delta V$ can be transformed into the cantilever deflection $z$, in $n m$, and the latter deflection can then be transformed into a force, in $\mathrm{nN}$ :

$$
\begin{aligned}
& z=S \Delta V \\
& F=k_{\text {eff }} z=k S \Delta V
\end{aligned}
$$

The tip-sample distance $d$ can be calculated as:

$$
d=d_{p}+z-d_{0}
$$

In Eq. 3, $\mathrm{d}_{p}$ decreases as the tip gets closer to the sample surface, and $z$ is positive when the cantilever is deflected upwards, under the action of a repulsive force, and negative in the opposite case. The parameter $\mathrm{d}_{0}$ represents the location along the $d_{p}+z$ axis where the tipsample distance is zero. The identification of $d_{0}$ is easy when the tip is ramped against a stiff substrate, since all data points belonging to the contact region of the force vs $d_{p}+z$ distance curve must collapse along a vertical line, whose corresponding mean abscissa value is $d_{0}$. On deformable surfaces, $\mathrm{d}_{0}$ is typically obtained through a fit of a suitable contact mechanics model (typically the Hertz model [51-53]) to the F vs $d_{p}+z$ curve [54].

The cantilever spring constant has been calibrated using the thermal noise method [43,44], and fine corrections were applied to account for geometrical and dimensional issues $[42,55]$. The deflection sensitivity $S$ of these probes was calculated according to different procedures: either as the inverse of the slope of the raw deflection $\Delta V$ vs z-piezo displacement $d_{p}$ curve (Figure 1B) acquired on a stiff substrate [47], or via the SNAP method [56], assuming a previously accurately calibrated intrinsic spring constant as reference.

After identification of $d_{0}$ and proper translation of the distance axis, negative distances correspond to deformations, i.e. indentations of the deformable sample. In nanomechanical tests, the negative semiaxis is the relevant one, and an indentation axis $\delta$ can be defined as: $\delta$ $=-d$, for $d<0$.

Processing of the data was carried out using custom routines written in Matlab environment (Mathworks).

The precise alignment of AFM and optical images was possible using the Bruker MIRO software and allowed to choose the regions of interest for ECMs and cells. For the ECMs, the regions for measurements were chosen based on evaluation of optical images; thanks to the reduced thickness of the slices and their consequent transparency, it was possible to select regions with moderate roughness and better structural integrity. 


\section{Indentation of living cells and ECMs by AFM}

Cells. For the mechanical characterization of cells, the Hertz model was applied [51-53]. To extract the value of the Young's modulus (YM) $E$, which is the proportionality constant (within the limits of linear elastic response) of stress $\sigma$ (force per unit area, in $\mathrm{Pa}$ ) and strain $\varepsilon$ (relative deformation): $E=\sigma / \varepsilon$. The $\mathrm{YM}$ is an intrinsic elastic property of a material and provides a measure of sample rigidity. According to the Hertz model for a parabolic indenter, the force vs indentation relation is:

$$
F=\frac{4}{3} \frac{E}{1-v^{2}} R^{\frac{1}{2}} \delta^{\frac{3}{2}}
$$

which is accurate as long as the indentation $\delta$ is small compared to the radius $R$. In Eq. $4 v$ is the Poisson's coefficient, which is typically assumed to be equal to 0.5 for incompressible materials.

When indenting compliant thin cells (typically a few microns tall at their maximum height, i.e. above their nucleus), the finite-thickness effect must be taken into account. This effect is related to the influence of the stiff glass substrate underneath the cells, which confines the strain and stress fields and makes the elastic cell response stiffer, i.e. the measured Young's Modulus larger [53,57-60]. The finite-thickness correction depends on the ratio $\chi$ of the contact radius $a=\sqrt{R \delta}$ to the sample thickness $h$ (and not trivially on the ratio $\delta / h$ ):

$\chi=\frac{\sqrt{R \delta}}{h}$

Noteworthy, AFM provides the unique capability of measuring simultaneously both height and elastic properties of a sample (combining topographic and mechanical imaging [53]), therefore allowing to implement point by point corrections that depends on ratios like the one reported in Eq. 5, as for the present work.

A polynomial correction factor $\Delta(\chi)$ can be applied to the Hertz equation (Eq. 4), under the hypothesis that cells are partially bound to the substrate, and this allows to extract correct YM values irrespective to the local thickness of the sample. Following Dimitriadis et al. work [53,57]:

$\Delta(\chi)=1+1,009 \chi+1,032 \chi^{2}+0,578 \chi^{3}+0,051 \chi^{4}$

Introducing the rescaled force $F^{\prime}(\delta)=F(\delta) / \Delta(\chi(\delta))$, Eq. 4 can be replaced by the formally similar Eq. 7:

$F^{\prime}=\frac{4}{3} \frac{E}{1-v^{2}} R^{\frac{1}{2}} \delta^{\frac{3}{2}}$

For the evaluation of the Young's modulus of single and clustered cells, at least 10 cells, each from three to five petri dishes, were measured. For each measurement, FCs on both the substrate and the cells were acquired with a minimum of $10 \mathrm{FCs}$ on the surrounding substrate and 100 FCs on the cell; this allowed to calculate the local height of each single cell[53]. For full mapping of single cells/clusters, FCs were collected on a grid spanning an area of up to 100 $\mu \mathrm{m} \times 100 \mu \mathrm{m}$ around the cells, including both cells and substrate. Each FC contained 8192 
points, with ramp length $I=15 \mu \mathrm{m}$, maximum load $F_{\max }=5-10 \mathrm{nN}$, ramp frequency $f=1 \mathrm{~Hz}$. The probe radius was $R=5,7 \mu \mathrm{m}$ or $R=6,4 \mu \mathrm{m}$. Typically, indentation up to $2 \mu \mathrm{m}$ was achieved. We created masks based on the obtained topographic maps to select force curves belonging to distinct regions: the nuclear region and its complement, which is the union of cell perinuclear and peripheral regions $[53,60]$.

The same data were used for both mechanical analysis of cells and glycocalyx characterisation. The first $10 \%$ of the indentation range after the contact point is usually attributed to the contribution of the glycocalyx $[21,27,31,61,62]$. The YM of the cells was extracted by fitting the Hertz model to the FCs in a suitable sub-interval of the remaining $10-90 \%$ indentation, typically identified as the range where the value of $E$ does not change with indentation (Figure 2).

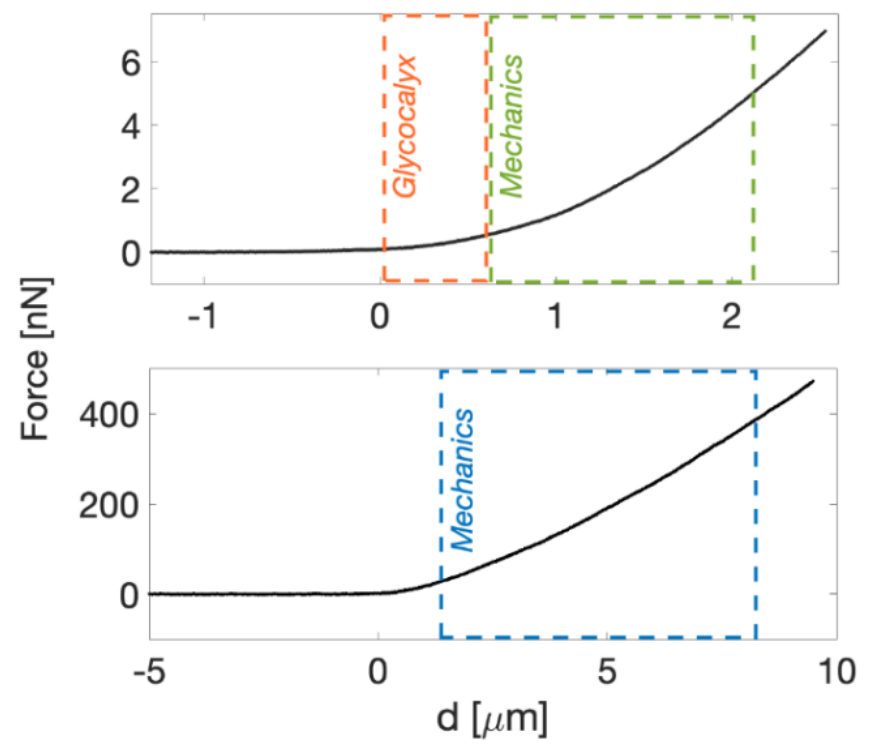

Figure 2. Typical approaching force curve on top of a cell (top) and ECM (bottom). The indentation range for model fitting are highlighted; on cells, we used typically [0-10\%] for the glycocalyx and [10$80 \%$ ] for the YM, while on ECMs we used [20-80\%] for the Young's modulus.

Glycocalyx thickness. The glycocalyx characterisation was performed following the soft brush model implemented by Sokolov et al.[27,31,61,63]. The separation $H$ between the tip and the cell membrane can be expressed as (Figure 3A):

$H=d_{p}-d_{0}+\delta+z$

, where $d_{0}$ is the position of the non-deformed cell membrane (the contact point referred to Hertzian indentation), $d_{p}$ is the relative z-piezo position, $\delta$ and $z$ are indentation and cantilever deflection, respectively.

The indentation $\delta$ in Eq. 8 is calculated using the standard Hertz model (Eq. 4). When the glycocalyx is completely compressed (which typically occurs well before cell indentation is significant), $H$ is negligibly small; it follows that if one plots the force as a function of $H$, the force points related to the Hertzian indentation of the cell collapse along a vertical line at $H \cong$ 
0 ; this can be seen in Figure 3B, where the force for $H>0$ can be identified with $F_{g l y c o c a l y x}$, the force exerted by the glycocalyx. The latter force can be modelled as [64] ${ }^{1}$ :

$$
F_{\text {glycocalyx }}=100 k_{B} T R N^{\frac{3}{2}} \exp \left(-\frac{2 \pi}{L} H\right) L
$$

, where $L$ and $N$ are the effective thickness and the grafting density of the pericellular brush, respectively.

A

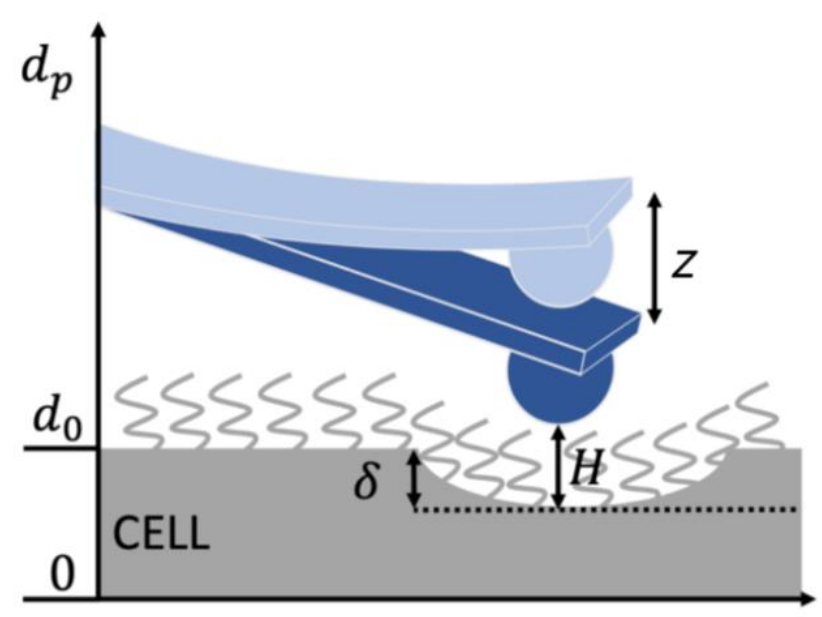

B

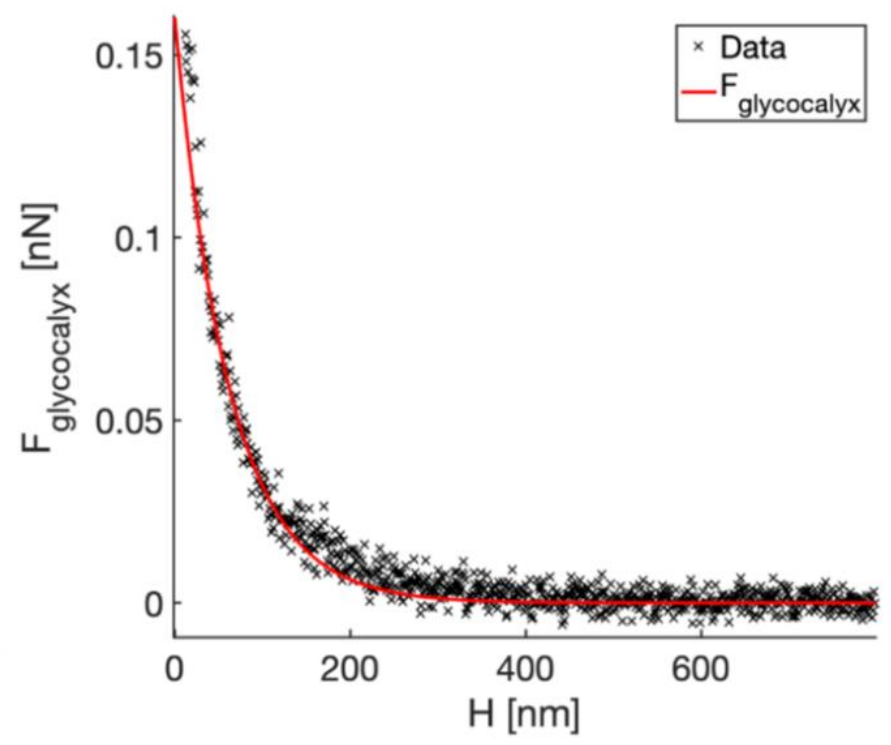

Figure 3. (A) Schematics of the distances used to determine the tip-cell membrane distance $H$ (Eq.8). (B) A typical force curve showing the force exerted by the glycocalyx as a function of the tip-cell membrane distance (the red continuous curve is the fit by Eq. 9).

ECMs. The mechanical properties of ECMs were studied by collecting sets of typically $15 \times 15$ force curves (force volumes, FV) in different macroscopically separated regions of the sample. Each selected region was typically as large as $115 \mu \mathrm{m} \times 115 \mu \mathrm{m}$. Each FC contained 8192 points, with ramp length $L=15 \mu \mathrm{m}$, maximum load $F_{\max }=800-1500 \mathrm{nN}^{2}$, ramp frequency $\mathrm{f}=1 \mathrm{~Hz}$ and $R=12,8 \mu \mathrm{m}$. Typical maximum indentation was 5-9 $\mu \mathrm{m}$. For each patient condition, 20005000 FCs were obtained.

The value of the YM of elasticity of ECM was extracted as described previously for cells. The Hertz model was fitted to the [20-80\%] indentation range of the FCs (Figure 2), without the finite-thickness correction (given the large thickness of the slices, $\chi \leq 0,1$ ). On tissues and ECMs, the first $20 \%$ of the FCs is typically neglected, due to the contribution of superficial noncrosslinked fibbers, surface roughness issues, etc. [5]

\footnotetext{
${ }^{1}$ Some authors use a prefactor of 50 instead of 100, as in Eq. 9. This should not impact on the accuracy of the determination of the glycocalyx thickness $L$.

2 Due to the very large radius of the colloidal probe, high force must be applied in order to achieve the desired indentation.
} 


\section{Statistics}

For both cells and ECM, the mean median value $\bar{E}_{\text {med }}$ of the Young's modulus $E$ (or the mean values of other observables) has been evaluated for each tested condition, averaging over cells or measured ECM samples. The associated errors were calculated adding in quadrature to the standard deviation of the mean $\sigma_{\bar{E}_{m e d}}$ an instrumental error of 3\%, calculated through a Monte Carlo simulation, as described in Refs $[53,66]$, based on the uncertainties in the calibration parameters ( $5 \%$ for the deflection sensitivity $S, 10 \%$ for the spring constant $k$ ).

The assessment of the statistical significance of the differences among the tested conditions was carried out using the two-tailed t-test. A p-value $<0.05$ was considered statistically significant.

For the glycocalyx analysis, the length $L$ of the glycocalyx for each force curve located on the nucleus was extracted, by fitting Eq. 9 to the data, and the histograms of the logarithmic values were reported. Median values were calculated.

\section{Results and discussion}

\section{AFM at the microscale: mechanical properties of ECMs}

In this experiment we carried out mechanical measurements on healthy and neoplastic decellularized extracellular matrices coming from the same patient affected by CRCPM. We measured YM values of the samples at deep indentation with focus on their distribution.

The production of custom colloidal probes allows to tune both the spring constant $k$ of the cantilever and the sphere radius $R$, to match the typical length scale of tissues and ECMs, which is approximately $10-50 \mu \mathrm{m}$ (cf. Material and methods)
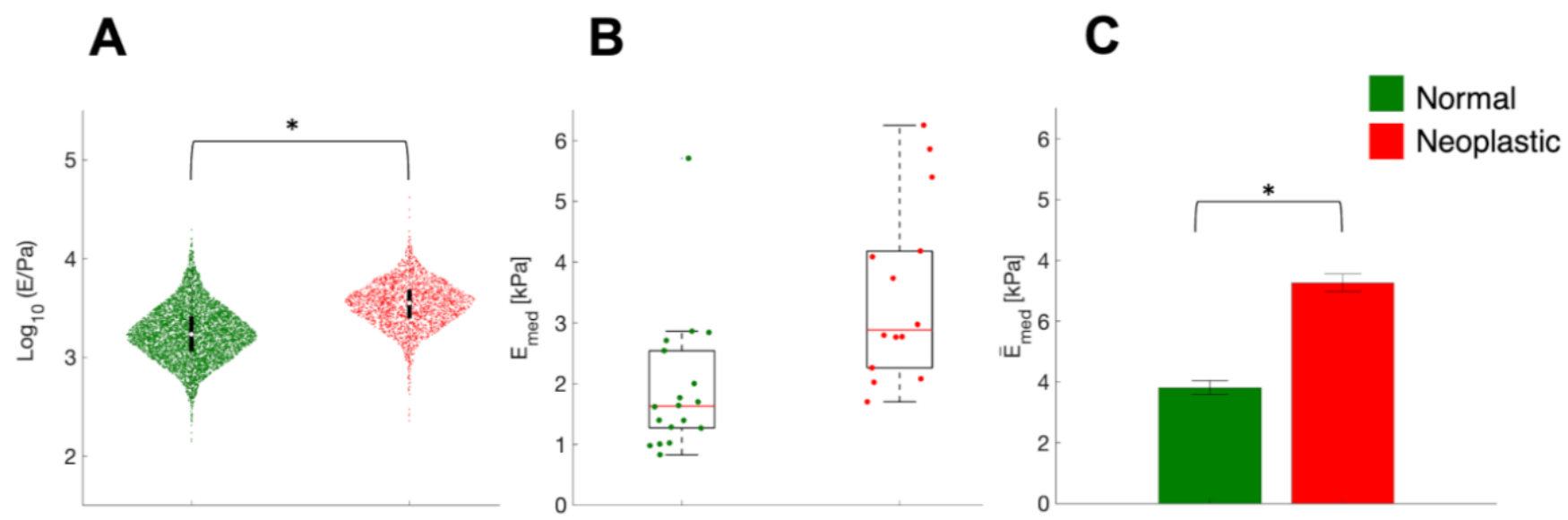

Figure 4. The stiffening of ECM in CRCPM samples. (A) Logarithmic values of the YM and their distribution in normal and neoplastic ECM samples obtained from one patient. Violin plot were plotted collecting YM values from all single FCs. Violin plots suggest that the distribution of local YM values is approximately lognormal. The circle and the black bars represent the median and the the interval between 25th and 75th percentiles. (B) Comparison of median YM values $E_{\text {med }}$ from each force volume in linear scale for normal and neoplastic samples from one patient. The red line represents the median value, the box encloses the interval between 25 th and 75 th percentiles of the sample. Whiskers go from the upper and lower limits of the interquartile range to the furthest observations, within $1.5 \mathrm{x}$ 
the interquartile range; data points beyond this limit are considered outliers. (C) Comparisn of the mean median $\mathrm{YM}$ values for the two conditions tested. In $\mathrm{A}$ and $\mathrm{C}, *$ means $\mathrm{p}<0.05$.

Exploiting large colloidal probe radius allows to effectively average local nanoscale heterogeneities due to the fine structure of the ECM, while capturing the overall mesoscopic mechanical response of the sample. To this purpose, it is important to achieve reasonably large (in absolute terms) indentations (5-9 $\mu \mathrm{m}$, compared to the 100-200 $\mu \mathrm{m}$ thickness of the samples). In these operative conditions, finite-thickness effects are negligible, and we are confident to test the bulk sample properties, as in a 3D structure, and not only those of a surface layer, which in similar samples can be different from the bulk. The measured mechanical response therefore reflects the collective contribution of all components of the ECM, organized in micrometre-sized structural and functional domains [5,17,67-71]. Small colloidal probes, and to a larger extent sharp pyramidal tips, would permit a greater spatial resolution, but the mechanical output would be more scattered and less representative of the overall properties of the ECM [53].

In Figure 4A, we show the distribution of the logarithmic YM values from each FCs taken on ECM samples. The fact that log YM values are approximately normally distributed suggests that the distribution of YM is lognormal, as it is typically observed [72].

During cancer progression, the neoplastic ECM becomes stiffer; indeed, the logarithmic YM distribution appears rigidly shifted to higher values (higher median value), while the logarithmic standard deviation is approximately preserved among normal and neoplastic conditions (Figure 3a). In Figure 4B, we show the distribution of the median YM values measured in different locations (FV) and slices of ECM are reported. Neoplastic-derived samples showed a significant increase of the stiffness, and this result was in line with data already published [1,2,9,73-75]; this stiffening during cancer progression is related to an outcome of the tumour microenvironment remodelling and changes in the ECM composition and structure, including aggregation and realignment of ECM components, mediated by tumoral cells [9].

\section{Mechanics of cell, down to cellular components.}

\section{A}

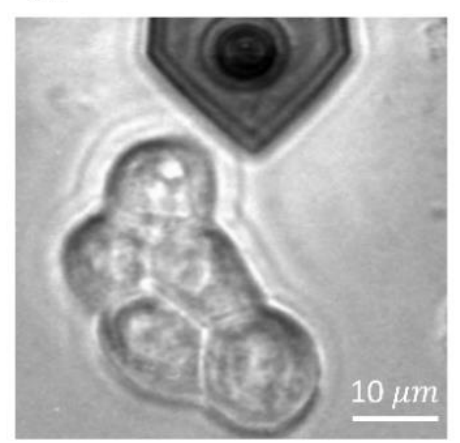

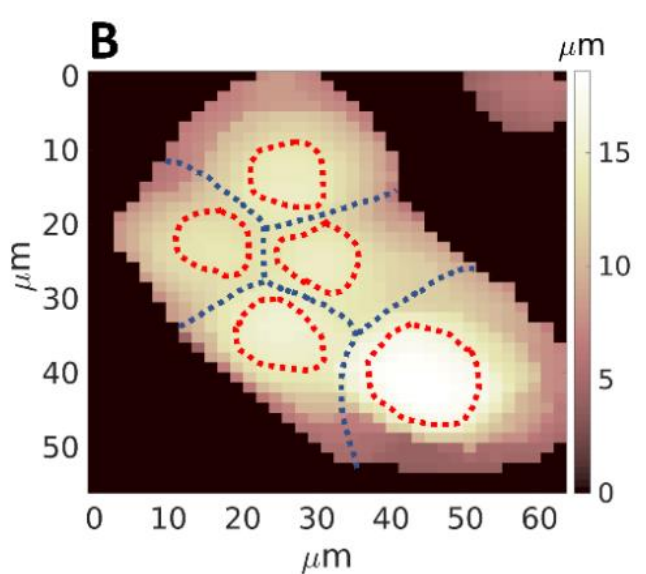

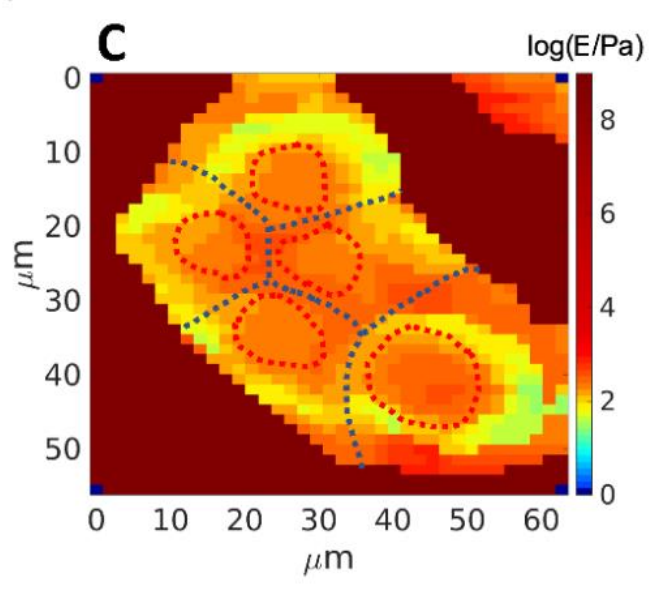


Figure 5. Representative images for the combined topographical and mechanical analysis of cell clusters. Optical image of a cell cluster from the RT112 cell line (A); topographic map (B), and Young's modulus map (C) in logarithmic scale, of the same cluster shown in (A).

Using AFM, it is also possible to sense small mechanical changes in single cells related to different physio-pathological conditions; using colloidal probes, the spatial resolution can be good enough to discriminate among single cell components, such as nuclear and perinuclear regions and lamellipodia [53], while sharp tips allow to discriminate fine cellular structures as small as single actin fibbers [76].

We performed AFM nanoindentation on three bladder cancer-derived cell lines, RT4, RT112 and T24, with different degree of invasiveness (Table 1), and compared their median YM values.

From the force vs distance curves, we reconstructed 3-dimensional cell morphologies and the mechanical maps, as described in Ref. [53]. All FCs, and consequently all maps, have been corrected for the contribution of the finite-thickness of the sample, as explained in the Methods.

Mapping both topography and YM and comparing with the optical image (Figure 5), allowed to decouple the contributions of the nuclear regions and the other regions of the cells in the same cluster. Examples of the distributions and median values of the YM extracted from different part of the cell body (e.g., nuclear vs perinuclear and peripheral regions, or lamellipodia) are shown in Figures 6,7.

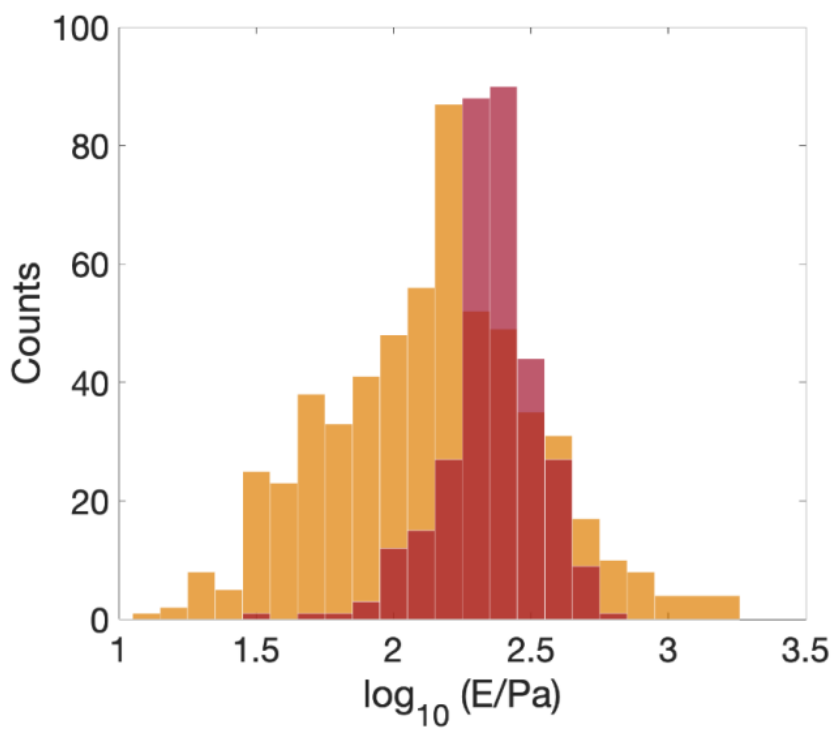

Figure 6. Histograms of the YM from the perinuclear and peripheral region of an RT112 cell (orange), and from the nuclear region (red). 


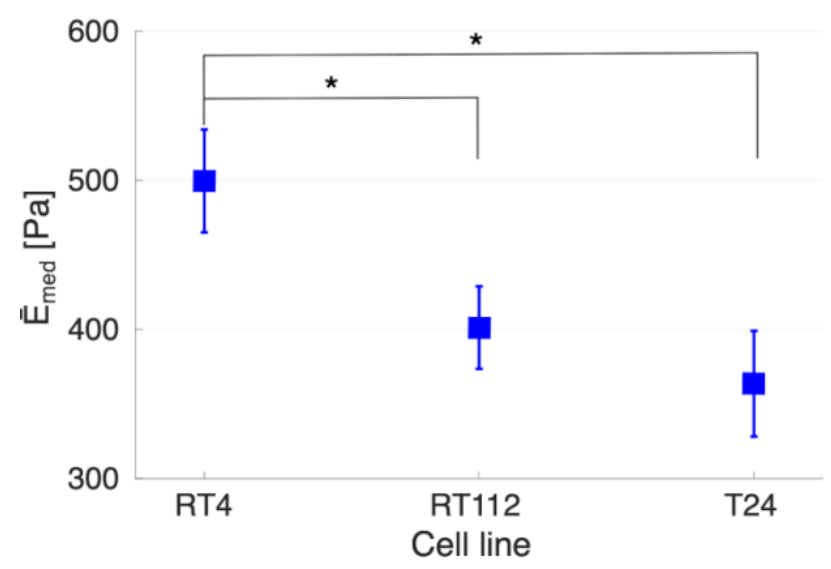

Figure 7. The Young's modulus (whole cell) measured by AFM for bladder cancer cells RT4, RT112 and T24, with increasing grade of invasiveness (left to right). ${ }^{*}$ means $p<0.05$.

As shown in Figure 7, the higher degree of invasiveness of cells (from RT4 to T24) correlates to a decrease of the YM (whole-cell value); this is consistent with previously published data [77]. The reported differences in YM between cell lines RT4 and RT112 and between RT4 and T24 were found to be significant, while this was not the case between RT112 and T24.

We observed a wider distribution of the YM values in the perinuclear and peripheral regions of the cells, compared to the nuclear region (Figure 6). In the perinuclear and peripheral regions, both softer and stiffer area coexist, as shown in Figure 5C; the higher Young's modulus values are found at the cell-cell boundaries, where adherent junctions are present. The nuclear region exhibits a narrower distribution of YM values (Figure 6), and is stiffer than the perinuclear region, as reported also previously $[19,78]$.

It is well known that during the embryonic and cancer development, cells exhibit a softening that can favour extravasating through the blood capillaries, allowing the attachment to a secondary site, favouring the metastatic spread in cancer[79]. The softening of the neoplastic cells has already been reported in breast and bladder cancer models [79-82]. It was reported that RT112 cells possess both mesenchymal and epithelial phenotype as they are an intermediate cell line for those states [11,83]. In our case, this correlates with the intermediate YM values observed for these cells compared to RT4 and T24.

\section{Down to the nanoscale: characterisation of the glycocalyx thickness}

As previously described, AFM is capable to sense the mechanical resistance to compression of tissue components like cells and ECM; it is possible to go further down along the size and force scales, characterising even smaller and more delicate structures such as the pericellular matrix, a sugar-rich coat, called glycocalyx.

Many models have been developed for the data analysis and the characterisation of the glycocalyx and similar brushes [27,84]. Here, we followed the protocol developed by Sokolov et al.[21,27,31,63], which is based on decoupling the deformation of the ultrasoft glycocalyx layer on top of the soft cell, within the acquired FCs. We applied this model to the FCs collected for the nanomechanical measurement of bladder cancer cells, to prove the feasibility of extracting more information from the same data set (see Materials and Methods).

To better appreciate the subtle differences between the three cell lines RT4, RT112 and T24, we considered the distribution of the glycocalyx thickness values extracted from the single FCs 
in log scale (Figure 8). There are differences in the distributions of $L$ values in the three cases. Compared to the intermediate grade of invasiveness (RT112), the less and most invasive cells (RT4 and T24, respectively) possess a broader distribution of brush lengths, with tails extending towards longer and shorter lengths, respectively; the median values of the glycocalyx thickness therefore tends to decrease going toward higher degree of invasiveness, from $L=730 \mathrm{~nm}$ (RT4 and RT112) to $L=652$ (T24). Nevertheless, the distribution seems to possess different modes (highlighted by the dotted vertical lines in Figure 8), and one can see that the relative importance of higher-thickness modes increases for more invasive cells, which are also characterised by the more asymmetric brush length distribution, as it was observed for tumoral cells $[25,30]$. These data suggest that beside the mere thickness/length of the brush, also the change of other glycocalyx physical properties, such as the stiffness, the effective graft density and degree of crosslinking, should be quantitatively characterized, since they are likely correlated to the transformation of a tissue from normal to neoplastic condition.
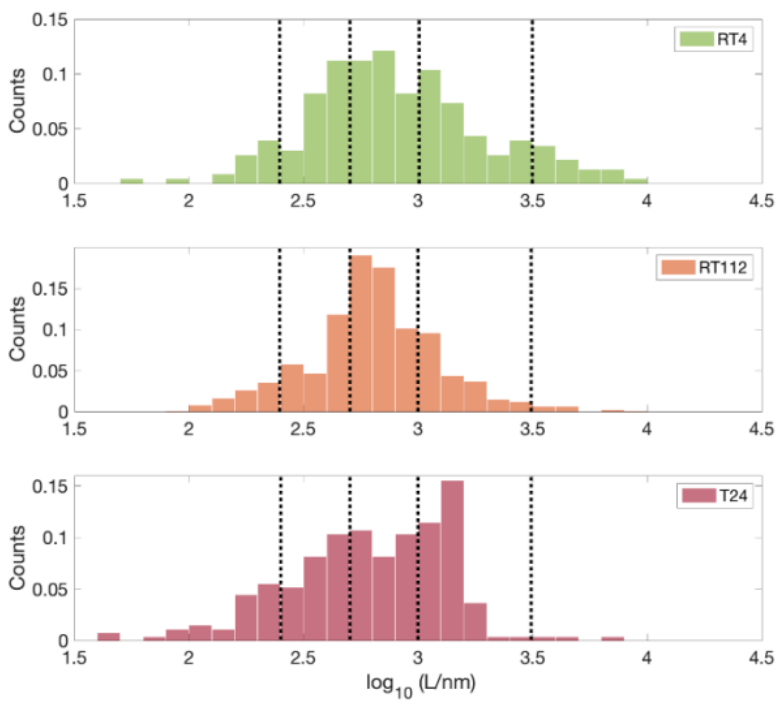

Figure 8. Distribution of the lengths of the glycocalyx brush (extracted according to Eq.9 from single FCs) for the three cell lines RT4, RT112 and T24. Vertical dotted lines are a guide for the eye in the tentative identification of the main modes of the distributions.

\section{Conclusions}

In this work, we discussed the capability of the AFM as a reliable force sensor in biological systems, to characterise physical modifications related to specific physio-pathological conditions of cells and tissues. Our results show the possibility of force-sensing with Atomic Force Microscopy on a variety of biological sample using nanomechanical measurements.

As demonstrated in this work, as well as in many others [36,85-88] , AFM can be used to test biological samples at several different scales, in terms of dimensions and forces, from large, rough and relatively stiff ECMs and tissues, passing through smaller and soft cells, to extremely compliant pericellular brushes. Together, our data demonstrate the capabilities of the instrument to characterise the mechanical differences of urothelium bladder cancer cells and the different organisation of their glycocalyx brushes using the same mechanical data set. On 
a larger scale, the stiffening of human-derived ECM during the progression of colorectal carcinoma could be detected.

The crosstalk between cells and their microenvironment is complex and challenging to be quantitatively assessed; the reliability of AFM also stands in its flexibility of measurements, which is demonstrated by the capability of AFM of both sensing and applying forces in aqueous physiological conditions, with controlled temperature, as well as by the possibility of resolving the measurements not only spatially, but also in the time and frequency domains.

AFM and AFM-inspired instruments will likely play an increasingly important role in establishing experimental approaches for the mechanical phenotyping of cells and tissues in health and disease conditions, with the potential of developing effective early diagnostic tools based on biomechanical measurements.

\section{Acknowledgements}

We thank Dr. Massimo Alfano (San Raffaele Hospital, Milano) for kindly providing the bladder cancer cell lines used in this study. We acknowledge the support of the European Union's Horizon 2020 research and innovation programme under the Marie Skłodowska-Curie grant agreement No. 812772, project Phys2Biomed, and under FET Open grant agreement No. 801126 , project EDIT. We thank the patients who participated to the study.

\section{References}

1. Frantz, C.; Stewart, K.M.; Weaver, V.M. The Extracellular Matrix at a Glance. Journal of Cell Science 2010, 123, 4195-4200, doi:10.1242/jcs.023820.

2. Cox, T.R. The Matrix in Cancer. Nature Reviews Cancer 2021, 21, 217-238, doi:10.1038/s41568-020-00329-7.

3. Winkler, J.; Abisoye-Ogunniyan, A.; Metcalf, K.J.; Werb, Z. Concepts of Extracellular Matrix Remodelling in Tumour Progression and Metastasis. Nature Communications 2020, 11, 5120, doi:10.1038/s41467-020-18794-x.

4. Engler, A.J.; Sen, S.; Sweeney, H.L.; Discher, D.E. Matrix Elasticity Directs Stem Cell Lineage Specification. Cell 2006, 126, 677-689, doi:10.1016/j.cell.2006.06.044.

5. Nebuloni, M.; Albarello, L.; Andolfo, A.; Magagnotti, C.; Genovese, L.; Locatelli, I.; Tonon, G.; Longhi, E.; Zerbi, P.; Allevi, R.; et al. Insight on Colorectal Carcinoma Infiltration by Studying Perilesional Extracellular Matrix. Scientific Reports 2016, 6, 22522, doi:10.1038/srep22522.

6. Lu, P.; Weaver, V.M.; Werb, Z. The Extracellular Matrix: A Dynamic Niche in Cancer Progression. The Journal of cell biology 2012, 196, 395-406, doi:10.1083/JCB.201102147.

7. Viji Babu, P.K.; Radmacher, M. Mechanics of Brain Tissues Studied by Atomic Force Microscopy: A Perspective. Frontiers in Neuroscience 2019, 13, doi:10.3389/fnins.2019.00600.

8. Muiznieks, L.D.; Keeley, F.W. Molecular Assembly and Mechanical Properties of the Extracellular Matrix: A Fibrous Protein Perspective. Biochimica et Biophysica Acta (BBA) - Molecular Basis of Disease 2013, 1832, 866-875, doi:10.1016/j.bbadis.2012.11.022.

9. Najafi, M.; Farhood, B.; Mortezaee, K. Extracellular Matrix (ECM) Stiffness and Degradation as Cancer Drivers. Journal of Cellular Biochemistry 2019, 120, 2782-2790, doi:10.1002/jcb.27681. 
10. Oxnard, G.R. The Cellular Origins of Drug Resistance in Cancer. Nature Medicine 2016, 22, 232-234, doi:10.1038/nm.4058.

11. Giroldi, L.A.; Bringuier, P.-P.; Shimazui, T.; Jansen, K.; Schalken, J.A. Changes in Cadherins-Catenin Complexes in the Progression of Human Bladder Carcinoma. J. Cancer 1999, 82, 70-76, doi:10.1002/(SICI)1097-0215(19990702)82:1.

12. Yeh, Y.-T.; Hur, S.S.; Chang, J.; Wang, K.-C.; Chiu, J.-J.; Li, Y.-S.; Chien, S. Matrix Stiffness Regulates Endothelial Cell Proliferation through Septin 9. PLOS ONE 2012, 7, e46889, doi:10.1371/journal.pone.0046889.

13. Jaalouk, D.E.; Lammerding, J. Mechanotransduction Gone Awry. Nature Reviews Molecular Cell Biology 2009, 10, 63-73, doi:10.1038/nrm2597.

14. Bissell, M.J.; Hines, W.C. Why Don't We Get More Cancer? A Proposed Role of the Microenvironment in Restraining Cancer Progression. Nature Medicine 2011, 17, 320329.

15. Chaudhuri, O.; Koshy, S.T.; Branco Da Cunha, C.; Shin, J.W.; Verbeke, C.S.; Allison, K.H.; Mooney, D.J. Extracellular Matrix Stiffness and Composition Jointly Regulate the Induction of Malignant Phenotypes in Mammary Epithelium. Nature Materials 2014, 13, 970-978, doi:10.1038/nmat4009.

16. Alberts, Bruce. Essential Cell Biology; 2nd ed.; Garland Science Pub.: New York NY, 1998; Vol. 51; ISBN 9780815334804.

17. Lansky, Z.; Mutsafi, Y.; Houben, L.; Ilani, T.; Armony, G.; Wolf, S.G.; Fass, D. 3D Mapping of Native Extracellular Matrix Reveals Cellular Responses to the Microenvironment. Journal of Structural Biology: X 2019, 1, 100002, doi:10.1016/j.yjsbx.2018.100002.

18. Alcaraz, J.; Otero, J.; Jorba, I.; Navajas, D. Bidirectional Mechanobiology between Cells and Their Local Extracellular Matrix Probed by Atomic Force Microscopy. Seminars in Cell and Developmental Biology 2018, 73, 71-81, doi:10.1016/j.semcdb.2017.07.020.

19. Liu, H.; Wen, J.; Xiao, Y.; Liu, J.; Hopyan, S.; Radisic, M.; Simmons, C.A.; Sun, Y. In Situ Mechanical Characterization of the Cell Nucleus by Atomic Force Microscopy. ACS Nano 2014, 8, 3821-3828, doi:10.1021/nn500553z.

20. Gao, L.; Lipowsky Herbert H., H.H. Composition of the Endothelial Glycocalyx and Its Relation to Its Thickness and Diffusion of Small Solutes. Microvascular Research 2010, 80, 394-401, doi:10.1016/j.mvr.2010.06.005.

21. Chighizola F, M.; Dini, T.; Marcotti, S.; Urso, M.D.'; Piazzoni, C.; Borghi, F.; Previdi, A.; Ceriani, L.; Folliero, C.; Stramer, B.; et al. The Glycocalyx Affects Force LoadingDependent Mechanotransductive Topography Sensing at the Nanoscale. bioRxiv 2021, 2021.03.02.433591, doi:10.1101/2021.03.02.433591.

22. Fu, B.M.; Tarbell, J.M. Mechano-Sensing and Transduction by Endothelial Surface Glycocalyx: Composition, Structure, and Function. Wiley Interdisciplinary Reviews: Systems Biology and Medicine 2013, 5, 381-390, doi:10.1002/wsbm.1211.

23. Kechagia, J.Z.; Ivaska, J.; Roca-Cusachs, P. Integrins as Biomechanical Sensors of the Microenvironment. Nature Reviews Molecular Cell Biology 2019, 20, 457-473, doi:10.1038/s41580-019-0134-2.

24. Paszek, M.J.; Boettiger, D.; Weaver, V.M.; Hammer, D.A. Integrin Clustering Is Driven by Mechanical Resistance from the Glycocalyx and the Substrate. PLoS Computational Biology 2009, 5, e1000604, doi:10.1371/journal.pcbi.1000604.

25. Paszek, M.J.; Dufort, C.C.; Rossier, O.; Bainer, R.; Mouw, J.K.; Godula, K.; Hudak, J.E.; Lakins, J.N.; Wijekoon, A.C.; Cassereau, L.; et al. The Cancer Glycocalyx Mechanically 
Primes Integrin-Mediated Growth and Survival. Nature 2014, 511, 319-325, doi:10.1038/nature13535.

26. Cosgun, Z.C.; Fels, B.; Kusche-Vihrog, K. Nanomechanics of the Endothelial Glycocalyx: From Structure to Function. American Journal of Pathology 2020, 190, 732-741, doi:10.1016/j.ajpath.2019.07.021.

27. Iyer, S.; Gaikwad, R.M.; Subba-Rao, V.; Woodworth, C.D.; Sokolov, I. Atomic Force Microscopy Detects Differences in the Surface Brush of Normal and Cancerous Cells. Nature Nanotechnology 2009, 4, 389-393, doi:10.1038/nnano.2009.77.

28. Schmidt, S.; Weigelin, B.; te Riet, J.; te Boekhorst, V.; te Lindert, M.; Wijers-Rouw, M.; Lelli, B.; Rognoni, L.; Krause-Vortmeyer, M.; Messent, A.; et al. Glycocalyx-Mediated Cell Adhesion and Migration. bioRxiv 2020, 2020.06.12.149096, doi:10.1101/2020.06.12.149096.

29. Kuo, J.C.H.; Gandhi, J.G.; Zia, R.N.; Paszek, M.J. Physical Biology of the Cancer Cell Glycocalyx. Nature Physics 2018, 14, 658-669, doi:10.1038/s41567-018-0186-9.

30. Buffone, A.; Weaver, V.M. Don't Sugarcoat It: How Glycocalyx Composition Influences Cancer Progression. Journal of Cell Biology 2020, 219, doi:10.1083/jcb.201910070.

31. Sokolov, I.; Iyer, S.; Subba-Rao, V.; Gaikwad, R.M.; Woodworth, C.D. Detection of Surface Brush on Biological Cells in Vitro with Atomic Force Microscopy. Applied Physics Letters 2007, 91, 023902, doi:10.1063/1.2757104.

32. Tarbell, J.M.; Cancel, L.M. The Glycocalyx and Its Significance in Human Medicine. Journal of Internal Medicine 2016, 280, 97-113, doi:10.1111/joim.12465.

33. Mitchell, M.J.; King, M.R. Physical Biology in Cancer. 3. The Role of Cell Glycocalyx in Vascular Transport of Circulating Tumor Cells. American Journal of Physiology - Cell Physiology 2014, 306, C89-C97, doi:10.1152/ajpcell.00285.2013.

34. Qi, S.; Yi, C.; Yang, M. Biosensors Using Atomic Force Microscopes. In Encyclopedia of Microfluidics and Nanofluidics; Springer US: Boston, MA, 2008; pp. 106-114.

35. Zhou, G.; Zhang, B.; Tang, G.; Yu, X.F.; Galluzzi, M. Cells Nanomechanics by Atomic Force Microscopy: Focus on Interactions at Nanoscale. Advances in Physics: $X$ 2021, 6 , doi:10.1080/23746149.2020.1866668.

36. Alessandrini, A.; Facci, P. AFM: A Versatile Tool in Biophysics. Measurement Science and Technology 2005, 16, R65-R92, doi:10.1088/0957-0233/16/6/R01.

37. Müller, D.J.; Dumitru, A.C.; lo Giudice, C.; Gaub, H.E.; Hinterdorfer, P.; Hummer, G.; de Yoreo, J.J.; Dufrêne, Y.F.; Alsteens, D. Atomic Force Microscopy-Based Force Spectroscopy and Multiparametric Imaging of Biomolecular and Cellular Systems. Chemical Reviews 2021, 121, 11701-11725, doi:10.1021/acs.chemrev.0c00617.

38. Meyer, G.; Amer, N.M. Optical-Beam-Deflection Atomic Force Microscopy: The $\mathrm{NaCl}$ (001) Surface. Applied Physics Letters 1990, 56, 2100-2101, doi:10.1063/1.102985.

39. Putman, C.A.J.; de Grooth, B.G.; van Hulst, N.F.; Greve, J. A Theoretical Comparison between Interferometric and Optical Beam Deflection Technique for the Measurement of Cantilever Displacement in AFM. Ultramicroscopy 1992, 42, 1509-1513, doi:10.1016/0304-3991(92)90474-X.

40. Alexander, S.; Hellemans, L.; Marti, O.; Schneir, J.; Elings, V.; Hansma, P.K.; Longmire, M.; Gurley, J. An Atomic-Resolution Atomic-Force Microscope Implemented Using an Optical Lever. Journal of Applied Physics 1989, 65, 164-167, doi:10.1063/1.342563.

41. Erlandsson, R.; McClelland, G.M.; Mate, C.M.; Chiang, S. Atomic Force Microscopy Using Optical Interferometry. Journal of Vacuum Science \& Technology A: Vacuum, Surfaces, and Films 1988, 6, 266-270, doi:10.1116/1.575440. 
42. Chighizola, M.; Puricelli, L.; Bellon, L.; Podestà, A. Large Colloidal Probes for Atomic Force Microscopy: Fabrication and Calibration Issues. Journal of Molecular Recognition 2021, 34, e2879, doi:10.1002/jmr.2879.

43. Butt, H.J.; Jaschke, M.; H.-J., B.; M., J. Calculation of Thermal Noise in Atomic Force Microscopy. Nanotechnology 1995, 6, 1-7, doi:10.1088/0957-4484/6/1/001.

44. Hutter, J.L.; Bechhoefer, J. Calibration of Atomic-Force Microscope Tips. Review of Scientific Instruments 1993, 64, 1868-1873, doi:10.1063/1.1143970.

45. Viani, M.B.; Schäffer, T.E.; Chand, A.; Rief, M.; Gaub, H.E.; Hansma, P.K. Small Cantilevers for Force Spectroscopy of Single Molecules. Journal of Applied Physics 1999, 86, 2258-2262, doi:10.1063/1.371039.

46. Smith, D.P.E. Limits of Force Microscopy. Review of Scientific Instruments 1995, 66, 3191-3195, doi:10.1063/1.1145550.

47. Butt, H.J.; Cappella, B.; Kappl, M. Force Measurements with the Atomic Force Microscope: Technique, Interpretation and Applications. Surface Science Reports 2005, 59, 1-152, doi:10.1016/j.surfrep.2005.08.003.

48. Genovese, L.; Zawada, L.; Tosoni, A.; Ferri, A.; Zerbi, P.; Allevi, R.; Nebuloni, M.; Alfano, M. Cellular Localization, Invasion, and Turnover Are Differently Influenced by Healthy and Tumor-Derived Extracellular Matrix. Tissue Engineering - Part A 2014, 20, 20052018, doi:10.1089/ten.tea.2013.0588.

49. Varinelli, L.; Guaglio, M.; Brich, S.; Zanutto, S.; Belfiore, A.; Zanardi, F.; lannelli, F.; Oldani, A.; Costa, E.; Chighizola, M.; et al. Decellularized Normal and Tumor Scaffolds for Cancer Organoid Cultures as a Model of Colorectal Peritoneal Metastases Short Title: Engineered 3D-Models of the Peritoneal Metastatic Niche. 2021, doi:10.1101/2021.07.15.452437.

50. Indrieri, M.; Podestà, A.; Bongiorno, G.; Marchesi, D.; Milani, P. Adhesive-Free Colloidal Probes for Nanoscale Force Measurements: Production and Characterization. Review of Scientific Instruments 2011, 82, 023708, doi:10.1063/1.3553499.

51. Kontomaris, S.-V. The Hertz Model in AFM Nanoindentation Experiments: Applications in Biological Samples and Biomaterials. Micro and Nanosystems 2018, 10, 11-22, doi:10.2174/1876402910666180426114700.

52. Hertz, H. Ueber Die Berührung Fester Elastischer Körper. Journal fur die Reine und Angewandte Mathematik 1881, 1881, 156-171, doi:10.1515/crll.1882.92.156.

53. Puricelli, L.; Galluzzi, M.; Schulte, C.; Podestà, A.; Milani, P. Nanomechanical and Topographical Imaging of Living Cells by Atomic Force Microscopy with Colloidal Probes. Review of Scientific Instruments 2015, 86, 33705, doi:10.1063/1.4915896.

54. Gavara, N. Combined Strategies for Optimal Detection of the Contact Point in AFM Force-Indentation Curves Obtained on Thin Samples and Adherent Cells. Scientific Reports 2016, 6, doi:10.1038/srep21267.

55. Laurent, J.; Steinberger, A.; Bellon, L. Functionalized AFM Probes for Force Spectroscopy: Eigenmode Shapes and Stiffness Calibration through Thermal Noise Measurements. Nanotechnology 2013, 24, 225504, doi:10.1088/09574484/24/22/225504.

56. Schillers, H.; Rianna, C.; Schäpe, J.; Luque, T.; Doschke, H.; Wälte, M.; Uriarte, J.J.; Campillo, N.; Michanetzis, G.P.A.; Bobrowska, J.; et al. Standardized Nanomechanical Atomic Force Microscopy Procedure (SNAP) for Measuring Soft and Biological Samples. Scientific Reports 2017, 7, doi:10.1038/s41598-017-05383-0. 
57. Dimitriadis, E.K.; Horkay, F.; Maresca, J.; Kachar, B.; Chadwick, R.S. Determination of Elastic Moduli of Thin Layers of Soft Material Using the Atomic Force Microscope. Biophysical Journal 2002, 82, 2798-2810, doi:10.1016/S0006-3495(02)75620-8.

58. Garcia, P.D.; Garcia, R. Determination of the Elastic Moduli of a Single Cell Cultured on a Rigid Support by Force Microscopy. Biophysical Journal 2018, 114, 2923-2932, doi:10.1016/j.bpj.2018.05.012.

59. Gavara, N.; Chadwick, R.S. Determination of the Elastic Moduli of Thin Samples and Adherent Cells Using Conical Atomic Force Microscope Tips. Nature Nanotechnology 2012, 7, 733-736, doi:10.1038/nnano.2012.163.

60. Kubiak, A.; Chighizola, M.; Schulte, C.; Bryniarska, N.; Wesolowska, J.; Pudelek, M.; Lasota, M.; Ryszawy, D.; Basta-Kaim, A.; Laidler, P.; et al. Stiffening of DU145 Prostate Cancer Cells Driven by Actin Filaments-Microtubule Crosstalk Conferring Resistance to Microtubule-Targeting Drugs. Nanoscale 2021, 13, 6212-6226, doi:10.1039/d0nr06464e.

61. Dokukin, M.; Ablaeva, Y.; Kalaparthi, V.; Seluanov, A.; Gorbunova, V.; Sokolov, I. Pericellular Brush and Mechanics of Guinea Pig Fibroblast Cells Studied with AFM. Biophysical Journal 2016, 111, 236-246, doi:10.1016/j.bpj.2016.06.005.

62. Giergiel, M.; Malek-Zietek, K.E.; Konior, J.; Targosz-Korecka, M. Endothelial Glycocalyx Detection and Characterization by Means of Atomic Force Spectroscopy: Comparison of Various Data Analysis Approaches. Micron 2021, 151, 103153, doi:10.1016/j.micron.2021.103153.

63. Sokolov, I.; Dokukin, M.E.; Guz, N. v. Method for Quantitative Measurements of the Elastic Modulus of Biological Cells in AFM Indentation Experiments. Methods 2013, 60, 202-213, doi:10.1016/j.ymeth.2013.03.037.

64. Dokukin, M.E.; Kuroki, H.; Minko, S.; Sokolov, I. AFM Study of Polymer Brush Grafted to Deformable Surfaces: Quantitative Properties of the Brush and Substrate Mechanics. Macromolecules 2017, 50, 275-282, doi:10.1021/acs.macromol.6b02149.

65. Israelachvili, J. Intermolecular and Surface Forces; Elsevier Inc., 2011; ISBN 9780123751829.

66. Alper, J.S.; Gelb, R.I. Standard Errors and Confidence Intervals in Nonlinear Regression: Comparison of Monte Carlo and Parametric Statistics; 1990; Vol. 94;.

67. Viji Babu, P.K.; Rianna, C.; Mirastschijski, U.; Radmacher, M. Nano-Mechanical Mapping of Interdependent Cell and ECM Mechanics by AFM Force Spectroscopy. Scientific Reports 2019, 9, doi:10.1038/s41598-019-48566-7.

68. Jorba, I.; Beltrán, G.; Falcones, B.; Suki, B.; Farré, R.; García-Aznar, J.M.; Navajas, D. Nonlinear Elasticity of the Lung Extracellular Microenvironment Is Regulated by Macroscale Tissue Strain. Acta Biomaterialia 2019, 92, 265-276, doi:10.1016/j.actbio.2019.05.023.

69. Jorba, I.; Uriarte, J.J.; Campillo, N.; Farré, R.; Navajas, D. Probing Micromechanical Properties of the Extracellular Matrix of Soft Tissues by Atomic Force Microscopy. Journal of Cellular Physiology 2017, 232, 19-26, doi:10.1002/jcp.25420.

70. Luque, T.; Melo, E.; Garreta, E.; Cortiella, J.; Nichols, J.; Farré, R.; Navajas, D. Local Micromechanical Properties of Decellularized Lung Scaffolds Measured with Atomic Force Microscopy. Acta Biomaterialia 2013, 9, 6852-6859, doi:10.1016/j.actbio.2013.02.044. 
71. Liu, F.; Mih, J.D.; Shea, B.S.; Kho, A.T.; Sharif, A.S.; Tager, A.M.; Tschumperlin, D.J. Feedback Amplification of Fibrosis through Matrix Stiffening and COX-2 Suppression. Journal of Cell Biology 2010, 190, 693-706, doi:10.1083/jcb.201004082.

72. Limpert, E.; Stahel, W.A. Problems with Using the Normal Distribution - and Ways to Improve Quality and Efficiency of Data Analysis. PLOS ONE 2011, 6, e21403, doi:10.1371/JOURNAL.PONE.0021403.

73. Deville, S.S.; Cordes, N. The Extracellular, Cellular, and Nuclear Stiffness, a Trinity in the Cancer Resistome-A Review. Frontiers in Oncology 2019, 9, doi:10.3389/fonc.2019.01376.

74. Grasset, E.M.; Bertero, T.; Bozec, A.; Friard, J.; Bourget, I.; Pisano, S.; Lecacheur, M.; Maiel, M.; Bailleux, C.; Emelyanov, A.; et al. Matrix Stiffening and EGFR Cooperate to Promote the Collective Invasion of Cancer Cells. Cancer Research 2018, 78, 5229-5242, doi:10.1158/0008-5472.CAN-18-0601.

75. Handorf, A.M.; Zhou, Y.; Halanski, M.A.; Li, W.J. Tissue Stiffness Dictates Development, Homeostasis, and Disease Progression. Organogenesis 2015, 11, 1-15, doi:10.1080/15476278.2015.1019687.

76. Rotsch, C.; Radmacher, M. Drug-Induced Changes of Cytoskeletal Structure and Mechanics in Fibroblasts: An Atomic Force Microscopy Study. Biophysical Journal 2000, 78, 520-535, doi:10.1016/S0006-3495(00)76614-8.

77. Liu, H.; Tan, Q.; Geddie, W.R.; Jewett, M.A.S.; Phillips, N.; Ke, D.; Simmons, C.A.; Sun, Y. Biophysical Characterization of Bladder Cancer Cells with Different Metastatic Potential. Cell Biochemistry and Biophysics 2014, 68, 241-246, doi:10.1007/s12013013-9702-9.

78. Tang, G.; Galluzzi, M.; Zhang, B.; Shen, Y.-L.; Stadler, F.J. Biomechanical Heterogeneity of Living Cells: Comparison between Atomic Force Microscopy and Finite Element Simulation. Langmuir 2019, 35, 7578-7587, doi:10.1021/acs.langmuir.8b02211.

79. Ramos, J.R.; Pabijan, J.; Garcia, R.; Lekka, M. The Softening of Human Bladder Cancer Cells Happens at an Early Stage of the Malignancy Process. Beilstein Journal of Nanotechnology 2014, 5, 447-457, doi:10.3762/bjnano.5.52.

80. Xu, W.; Mezencev, R.; Kim, B.; Wang, L.; McDonald, J.; Sulchek, T. Cell Stiffness Is a Biomarker of the Metastatic Potential of Ovarian Cancer Cells. PLOS ONE 2012, 7, 46609, doi:10.1371/journal.pone.0046609.

81. Cross, S.E.; Jin, Y.S.; Rao, J.; Gimzewski, J.K. Nanomechanical Analysis of Cells from Cancer Patients. Nature Nanotechnology 2007, 2, 780-783, doi:10.1038/nnano.2007.388.

82. Plodinec, M.; Loparic, M.; Monnier, C.A.; Obermann, E.C.; Zanetti-Dallenbach, R.; Oertle, P.; Hyotyla, J.T.; Aebi, U.; Bentires-Alj, M.; Lim, R.Y.H.; et al. The Nanomechanical Signature of Breast Cancer. Nature Nanotechnology 2012, 7, 757-765, doi:10.1038/nnano.2012.167.

83. Mialhe, A.; Levacher, G.; Champelovier, P.; Martel, V.; Serres, M.; Knudsen, K.; Seigneurin, D. Expression of $\mathrm{E}_{-}, \mathrm{P}-, \mathrm{N}$-Cadherins and Catenins in Human Bladder Carcinoma Cell Lines. Journal of Urology 2000, 164, 826-835, doi:10.1097/00005392200009010-00057.

84. Oberleithner, H.; Peters, W.; Kusche-Vihrog, K.; Korte, S.; Schillers, H.; Kliche, K.; Oberleithner, K. Salt Overload Damages the Glycocalyx Sodium Barrier of Vascular Endothelium. Pflugers Archiv European Journal of Physiology 2011, 462, 519-528, doi:10.1007/s00424-011-0999-1. 
85. Gould, S.A.C.; Drake, B.; Prater, C.B.; Weisenhorn, A.L.; Manne, S.; Kelderman, G.L.; Butt, H.J.; Hansma, H.; Hansma, P.K.; Magonov, S.; et al. The Atomic Force Microscope: A Tool for Science and Industry; 1990; Vol. 33;.

86. Müller, D.J.; Dufrêne, Y.F. Atomic Force Microscopy as a Multifunctional Molecular Toolbox in Nanobiotechnology. Nature Nanotechnology 2008, 3, 261-269, doi:10.1038/nnano.2008.100.

87. Krieg, M.; Fläschner, G.; Alsteens, D.; Gaub, B.M.; Roos, W.H.; Wuite, G.J.L.; Gaub, H.E.; Gerber, C.; Dufrêne, Y.F.; Müller, D.J. Atomic Force Microscopy-Based Mechanobiology. Nature Reviews Physics 2019, 1, 41-57, doi:10.1038/s42254-018-0001-7.

88. Li, M.; Dang, D.; Liu, L.; Xi, N.; Wang, Y. Atomic Force Microscopy in Characterizing Cell Mechanics for Biomedical Applications: A Review. IEEE Transactions on Nanobioscience 2017, 16, 523-540, doi:10.1109/TNB.2017.2714462. 